\title{
Exclusive vector mesons and DVCS at HERA
}

\author{
Armen Bunyatyan a b \\ ${ }^{a}$ Max-Planck-Institut für Kernphysik, Heidelberg, Germany \\ ${ }^{\text {b}}$ Yerevan Physics Institute, Armenia
}

The latest results on exclusive vector meson production and the deeply virtual photon scattering (DVCS) from HERA are reviewed. The measurements are compared with pQCD models.

\section{Introduction}

Diffractive production of vector mesons (VM) or real photons at HERA $\gamma^{*} p \rightarrow V p$ (with $\left.V=\rho^{0}, \omega, \phi, J / \psi, \Psi^{\prime}, \Upsilon\right)$ have been extensively studied at HERA. The exclusive VM production at HERA spans the whole range from "soft" diffraction, described by Regge phenomenology, to "hard" diffraction, where the hard scales, such as the squared momentum exchanges $Q^{2}, t$ at the respective photon and proton vertices or the heavy VM mass, are involved and where the pQCD calculations can be applied.

Among the most striking expectations in this transition is the change of the logarithmic derivative $\delta$ of the cross section with respect to the $\gamma^{*} p$ center-of-mass energy $W$, from a value of about 0.2 in the soft regime [1] (represented by a soft Pomeron exchange diagram in Fig.1a) to 0.8 in the hard one (represented by a twogluon exchange diagram in Fig.1b), and the decrease of the exponential slope $b$ of the differential cross section with respect to the squaredfour-momentum transfer $t$, from a value of about $10 \mathrm{GeV}^{2}$ to an asymptotic value of about $5 \mathrm{GeV}^{2}$ when the virtuality $Q^{2}$ of the photon increases.

The QCD description of the diffractive process involves the exchange of several partons, e.g. a colour-singlet pair of gluons. When calculating the cross section of exclusive electroproduction of vector mesons, one needs information on the wave function of the initial virtual photon, the wave function of the produced vector meson, the $q \bar{q} p$ scattering amplitude, which requires the gluon density and the proton elastic form factor (see Fig.1b). While the PDFs extracted from the inclusive scattering process do not contain information about correlations between partons, the elastic VM production offers the possibility to learn more about these correlations, which are encoded in the generalized parton distributions (GPD). In particular the Deeply Virtual Compton Scattering (DVCS), $e p \rightarrow e p \gamma$, where a real photon is produced in diffractive scattering, is ideal for this study since it has no complications due to presence of the VM wave function in the final state description.

In the following the recent result of $\mathrm{H} 1$ and ZEUS experiments on exclusive vector meson production and DVCS are reviewed.

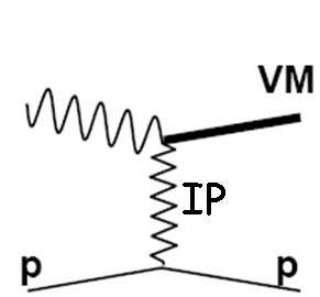

a)

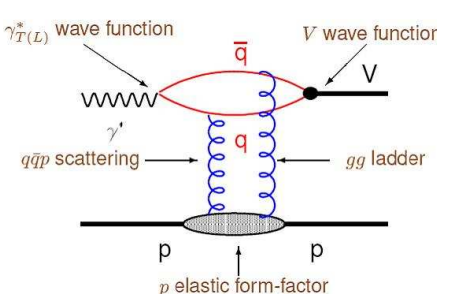

b)
Figure 1. a) A diagram describing exclusive vector meson production in terms of a Pomeron exchange. b) A diagram describing exclusive vector meson production in terms of a two gluon exchange. 


\section{Energy Dependence of the Cross Sec- tions}

The transition from the soft to the hard interactions is investigated by studying the energy dependence of the cross sections for exclusive photoproduction of vector mesons $\sigma(\gamma p \rightarrow V p)$, from the lightest one, $\rho^{0}$, to the heavier ones, up to the $\Upsilon[2-10]$. The results are shown in Fig.2. $W$ is the center-of-mass energy of the photon-proton system. For comparison, the total photoproduction cross section, $\sigma_{t o t}(\gamma p)[11,12]$, is also shown. The data at high $W$ can be parameterised as $W^{\delta}$, and the value of $\delta$ is displayed in the figure for each reaction. One sees clearly the transition from a shallow $W$ dependence for low scales, given by the mass of the vector meson, to a steeper one as the scale increases.

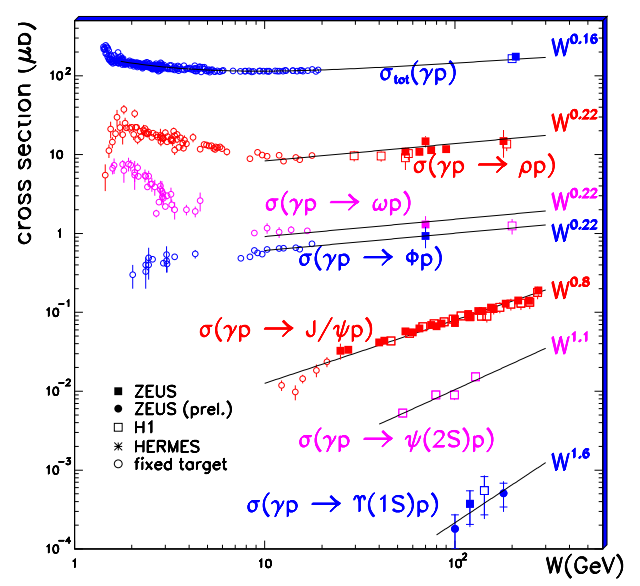

Figure 2. The cross section for diffractive vector meson production in the photoproduction kinematic regime $\left(Q^{2} \sim 0 \mathrm{GeV}^{2}\right)$. The results for various vector mesons are shown as a function of the center-of-mass energy of $\gamma p$ system- $W$, together with fits of the form $W^{\delta}$.

The transition from the soft to the hard interaction is also studied by varying the $Q^{2}$ for exclusive electroproduction of $\rho$ and $\phi$-mesons [13,14].
The cross section $\sigma\left(\gamma^{*} p \rightarrow \rho^{0} p\right)$ and $\sigma\left(\gamma^{*} p \rightarrow \phi p\right)$ are presented in Fig. 3 as a function of $W$, for different intervals of $Q^{2}$. The cross sections rise with $W$ for all $Q^{2}$. In order to quantify the rise, the logarithmic derivative $\delta$ of $\sigma$ with respect to $W$ is obtained by fitting the data to the expression $\sigma \sim W^{\delta}$ in each of the $Q^{2}$ intervals. The resulting values of $\delta$ for $\rho$-meson are shown in Fig. 4 - it is about 0.2 for photoproduction as expected for the soft interaction, and increases with increasing of hard scale $Q^{2}$ reaching a value of $\sim 0.8$ for the highest $Q^{2}$ value.

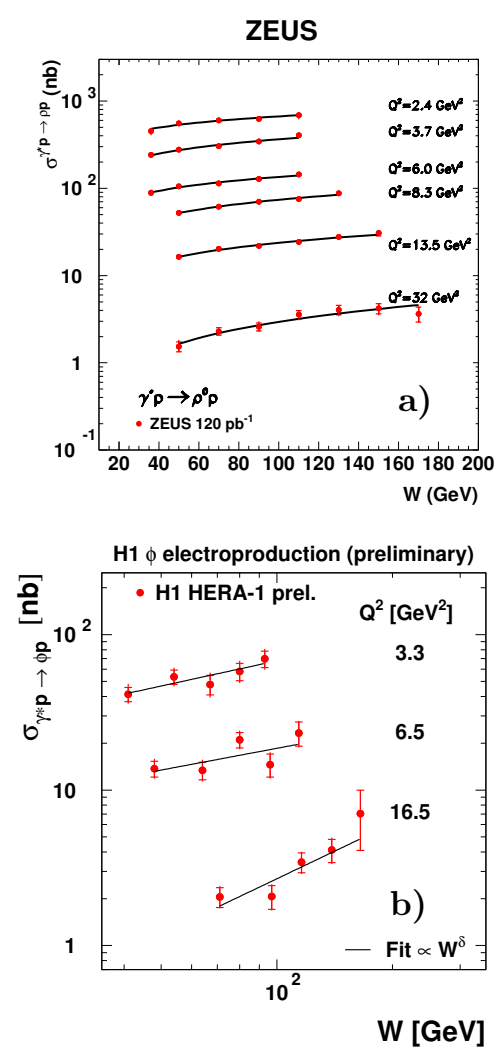

Figure 3. $W$ dependences of exclusive (a) $\rho$ - and (b) $\phi$-meson electroproduction for several $Q^{2}$ intervals.

Fig. 5 shows the similar distribution- $\delta$ vs $Q^{2}$ for elastic photo- and electroproduction of $\mathrm{J} / \psi$ $[3,15]$. For $J / \psi$-meson $\delta$ is almost independent 
ZEUS

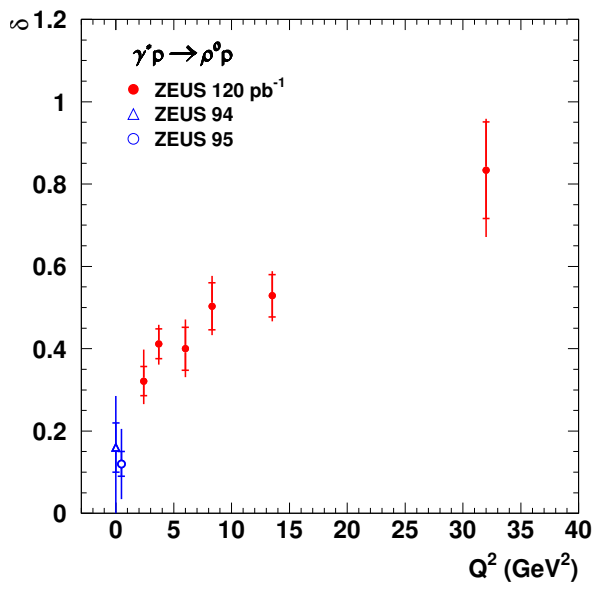

Figure 4. The values of $\delta$ from a fit of the form $\sigma \sim W^{\delta}$ for the exclusive electroproduction of $\rho^{0}$ mesons as a function of $Q^{2}$.

from $Q^{2}$ and has an average a value of 0.8 . Thus, the mass of $J / \psi$-meson plays the similar role of hard scale as $Q^{2}$ for the electroproduction of $\rho$ and $\phi$-mesons.

Fig. 6 shows the $W$ dependence of cross sections for the DVCS [16,17]. The cross sections show a steep rise for all $Q^{2}$ ranges, even for the lowest ones, and the value of $\delta$ is about 0.8 , almost independent of $Q^{2}$.

Fig. 7 shows the compilation of the values of $\delta$ for elastic photo- and electroproduction of $\rho, \phi$, $J / \psi$-meson and DVCS. The results are plotted as a function of $Q^{2}+M^{2}$, where $M$ is the mass of the vector meson. All these measurements seem to line up a universal curve. $\delta$ increases as the scale becomes larger, in agreement with the expectations. The values of $\delta$ at low scale is the one expected from the soft Pomeron intercept, while the one at large scale is in accordance with twice the logarithmic derivative of the gluon density with respect to $W$.

\section{3. $t$ Dependence of the Cross Section}

The differential cross sections, $d \sigma / d t$, have been measured for different $Q^{2}$ values for $\rho, \phi$, $J / \psi$-mesons and the DVCS [3,9,13-17]. The dis-

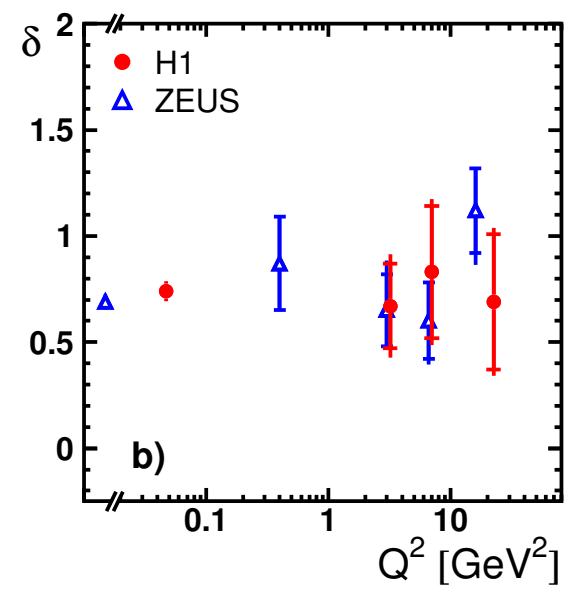

Figure 5. The value of $\delta$ from a fit of the form $\sigma \sim W^{\delta}$ for the elastic electroproduction of $J / \psi$-mesons $\left(\gamma^{*} p \rightarrow J / \psi p\right)$ as a function of $Q^{2}$.

tributions can be parameterised by an exponential function $e^{-b|t|}$ and the slope $b$ can be extracted from the fits. The resulting values of $b$ as a function of the scale $Q^{2}+M^{2}$ are shown in Fig.8. As expected, $b$ decreases to a universal value of about $5 \mathrm{GeV}^{-2}$ as the scale increases.

Assuming that the process of exclusive electroproduction of vector mesons is hard and dominated by gluons, $b$ is related to the radius of the gluon density in the proton. The values of about $5 \mathrm{GeV}^{-2}$ corresponds to a value of $\langle r\rangle_{g} \sim$ $0.6 \mathrm{fm}$, smaller than the value of the charge density of the proton $\left(<r>_{p} \sim 0.8 \mathrm{fm}\right)$, indicating that the gluons are well-contained within the charge-radius of the proton.

\section{Pomeron Trajectory}

The measurement of the $W$ dependence of $d \sigma / d t$ for fixed $t$ is used to extract the effective Pomeron trajectory $\alpha_{\mathbb{P}}(t)[14,18]$. The energy dependence of the cross section as a function of $t$ is parameterised with $\frac{d \sigma_{\gamma p}(W)}{d t}=$ $\frac{d \sigma_{\gamma p}\left(W_{0}\right)}{d t} \frac{W}{W_{0}}{ }^{4\left(\alpha_{\mathbb{P}}(t)-1\right)}$. The measurements of $\alpha_{\mathbb{P}}(t)$ for $\rho^{0}$ photoproduction is shown in Fig.9. The trajectory is fitted to a linear form to obtain the intercept $\alpha_{\mathbb{P}}(0)$ and the slope $\alpha_{\mathbb{P}}^{\prime}$. From the 

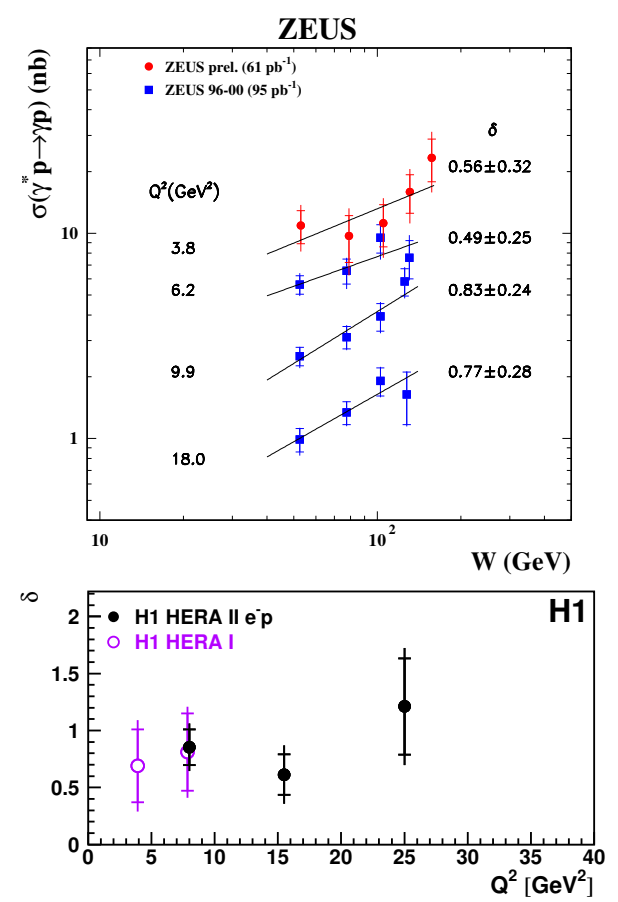

Figure 6. The DVCS cross section $\sigma\left(\gamma^{*} p \rightarrow \gamma p\right)$ as a function of $W$ for different intervals of $Q^{2}$. The lines are results of the fit of the form $\sigma \sim W^{\delta}$. The lower plot shows the value of $\delta$ from a fit as a function of $Q^{2}$.

fits the values of $\alpha_{\mathbb{P}}(0)=1.093 \pm 0.003_{-0.007}^{+0.008}(\mathrm{H} 1)$ and $1.096 \pm 0.021$ (ZEUS) and the values of $\alpha_{\mathbb{P}}^{\prime}=0.116 \pm 0.027_{-0.046}^{+0.036} \mathrm{GeV}^{-2}(\mathrm{H} 1)$ and $0.125 \pm$ $0.038 \mathrm{GeV}^{-2}$ (ZEUS) are obtained. The Pomeron intercept $\alpha_{\mathbb{P}}(0)$ is similar to the value $1.08 \mathrm{ex}$ pected for Regge trajectory for hadron-hadron collisions, while the the slope $\alpha_{I P}^{\prime}$ is almost twice smaller than expected value of $0.25 \mathrm{GeV}^{-2}$.

The $W$-dependences of elastic production of $\rho$, $\phi$ and $J / \psi$ - mesons are summarized in Fig.10 in the form of the $Q^{2}+M^{2}$ dependence of $\alpha_{\mathbb{P}}(0)$. A common increase of $\alpha_{\mathbb{P}}(0)$ with $Q^{2}+M^{2}$ is observed, related to the hardening of the gluon distribution with the hard scale of the interaction.

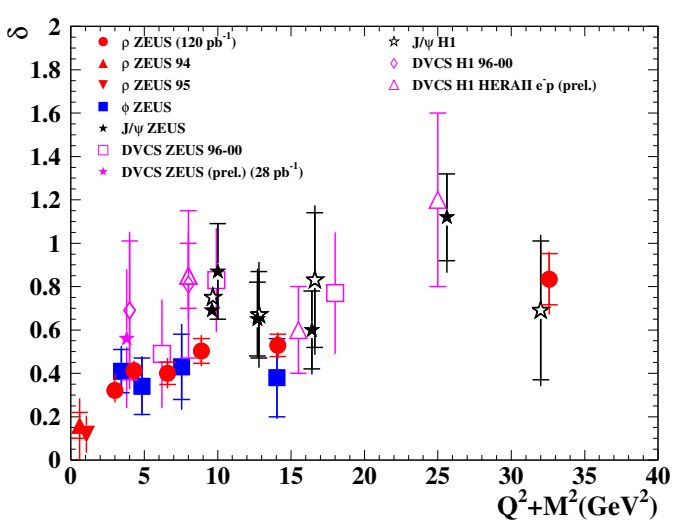

Figure 7. A compilation of the value of $\delta$ from a fit of the form $W^{\delta}$ for exclusive vector-meson electroproduction, as a function of $Q^{2}+M^{2}$.

\section{5. $Q^{2}, W$ and $t$ Dependence of $r_{00}^{04}=\sigma_{L} / \sigma_{t o t}$ for $\gamma^{*} p \rightarrow \rho^{0} p$}

The helicity analysis of the decay matrix element $r_{00}^{04}$ of the $\rho^{0}$ was used to extract the ratio $R=\sigma_{L} / \sigma_{T}$ of longitudinal $\left(\sigma_{L}\right)$ to transverse $\left(\sigma_{T}\right) \gamma^{*} p$ cross sections $[13,14]$. While the ratio is an increasing function of $Q^{2}$, as shown in Fig.11, it is independent of $W$ and $t$ in all $Q^{2}$ intervals (Fig.12). This implies that the $W$ and $t$ behavior of $\sigma_{L}$ is the same as that of $\sigma_{T}$. This result is not trivial. The $q \bar{q}$ configurations in the wave function of $\gamma_{L}^{*}$ have typically a small transverse size, while the configurations contributing to $\gamma_{T}^{*}$ may have large transverse sizes. The contribution to $\sigma_{T}$ of large-size $q \bar{q}$ configurations, which are more hadron like, is expected to lead to a shallower $W$ dependence than in case of $\sigma_{L}$. Different sizes of interacting objects also imply different $t$ distributions, in particular a steeper behavior for $\sigma_{T}$ compared to $\sigma_{L}$. This observation suggests that the large-size configurations of the transversely polarized photon are suppressed.

\section{Polarized Cross Sections}

The $\gamma^{*} p$ cross section can be expressed as the sum of the contributions of transversely and longitudinally polarised virtual photons. The lon- 


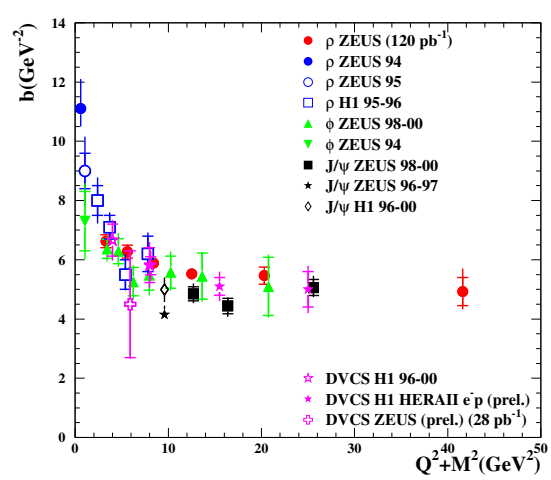

Figure 8. A compilation of the value of $b$-slope from a fit of the form $d \sigma / d t \sim e^{b|t|}$ for exclusive vector meson electroproduction, as a function of $Q^{2}+M^{2}$.

gitudinal and transverse $\gamma^{*} p$ cross sections are presented in Fig.13 for elastic and proton dissociative $\rho$ and $\phi$ production, using the total cross section measurements and the cross section ratio $R=\sigma_{L} / \sigma_{T}$ obtained from measurements of the spin density matrix elements. The polarised cross sections are compared with the model predictions. The GPD model [19] describes the measurements within the theoretical and experimental errors, the $k_{T}$ model [20] predictions are too steep. The dipole mode [21] describes relatively well the $\sigma_{L}$, bit for $\sigma_{T}$ it falls slightly too fast with $Q^{2}+M^{2}$.

\section{Comparison of the Data of Exclusive Electroproduction of Vector Mesons to Models}

The measurement of exclusive vector meson production reach the precision level, where they can contribute to a better understanding of the $\mathrm{VM}$ wave function and of the gluon density in the proton. The measurement of the process $\gamma^{*} p \rightarrow \rho^{0} p$ are compared to some selected pQCD inspired models. The models differ in the assumptions on wave functions, corrections applied to leading order calculations, assumptions on generalized parton distributions. The predictions are

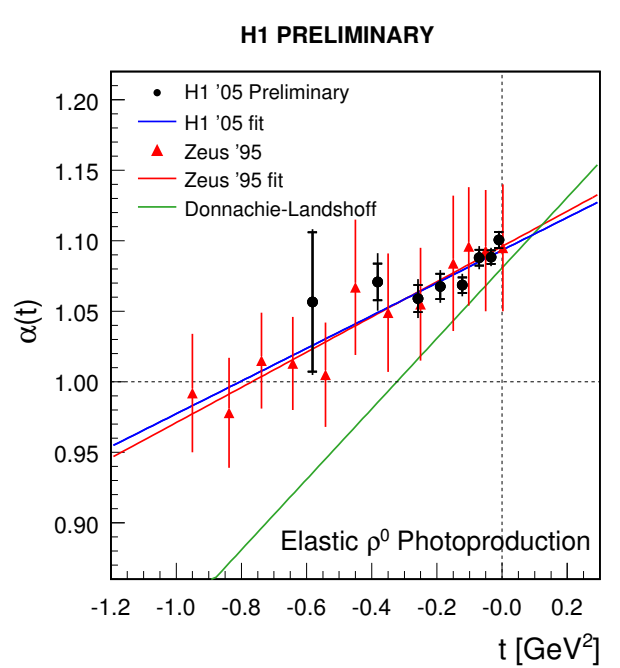

Figure 9. The effective Pomeron trajectory $\alpha_{\mathbb{P}}(t)$ measured in elastic $\rho^{0}$ photoproduction.

compared with the $W$-dependence of cross section for different $Q^{2}$ values in Fig.14. None of models reproduces the magnitude of the cross section measurements. The closest to the data, in shape and magnitude, are the MRT [22] model with the CTEQ6.5M parameterisation of the gluon distribution in the proton [23] and the KMW [24] model.

In Fig.15 the pQCD calculations of [22] are compared with the cross section of elastic $\mathrm{J} / \psi$ production as a function of $Q^{2}$ at $W_{\gamma p}=90 \mathrm{GeV}$. Results with four different gluon distributions are shown. The distribution is well described by a model within the theoretical and experimental uncertainties for some gluon parameterisations. This demonstrates the potential to constrain the gluon distribution in a kinematic range (low $x$, low $Q^{2}$ ) where the inclusive DIS data yield gluon distributions with large uncertainties.

\section{QCD Interpretation of DVCS Results in Terms of GPD}

To describe the DVCS process it is convenient to introduce two dimensionless observables: $S=\sqrt{\frac{\sigma_{D V C S} Q^{4} b\left(Q^{2}\right)}{\left(1+\rho^{2}\right)}}$ and $R=\frac{4 \sqrt{\pi \sigma_{D V C S} b\left(Q^{2}\right)}}{\sigma_{T}\left(\gamma^{*} p \rightarrow X\right) \sqrt{\left(1+\rho^{2}\right)}}$. 


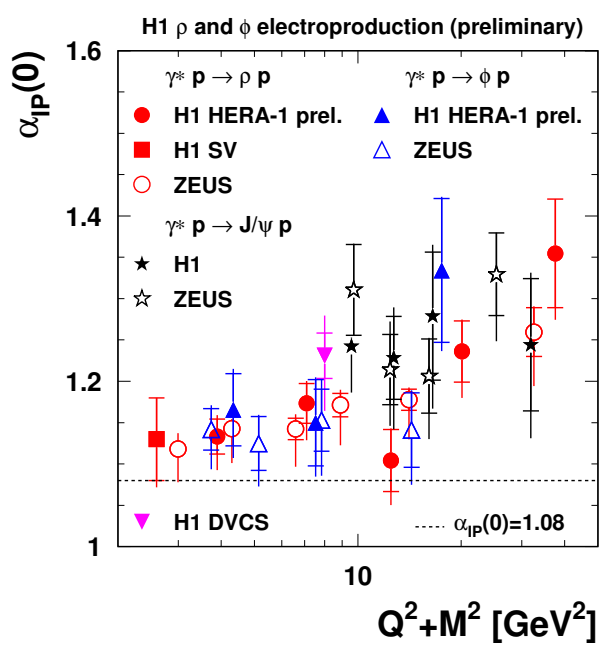

Figure 10. Value of the intercept of the effective Pomeron trajectory $\alpha_{\mathbb{P}}(0)$ as a function of $Q^{2}+$ $M^{2}$, as obtained from measurements of exclusive electroproduction of $\rho^{0}, \phi$ and $J / \psi$ vector mesons.

The observable $S$ gives access to the $Q^{2}$ evolution of GPD. The observable $R$ at leading order is equal to the ratio of the GPDs to the PDF, thus it provides the magnitude of the skewing effects present in the DVCS process. The measured values of $S$ and $R$ as function of $Q^{2}$ are shown in Fig.16 and compared with the calculation based on the GPD model [26], based on the PDF parameterisation given in [23]. It is observed that the pQCD skewed evolution equations provide a reasonable description of the measured weak rise of $S$ with $Q^{2}$. For the observable $R$, the typical values are around 2 , whereas in a model without skewing $R$ would be equal to unity. Therefore, the present measurement confirms the large effect of skewing.

\section{Measurement of Beam Charge Asym- metry in DVCS}

A direct access to the generalized parton distributions in the proton gives the measurement of

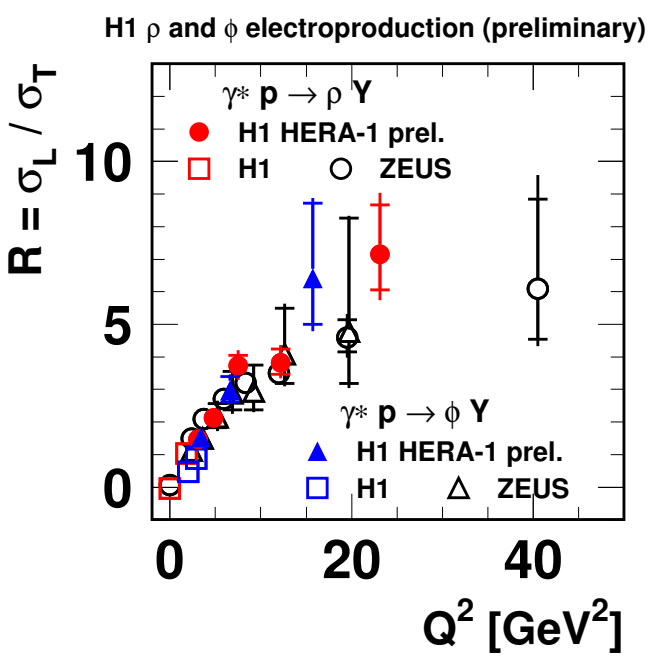

Figure 11. Ratio $R=\sigma_{L} / \sigma_{T}$ of the longitudinal and transverse cross sections for exclusive $\rho$ and $\phi$-meson electroproduction as a function of $Q^{2}$.

beam-charge asymmetry of DVCS. It is defined as

$$
B C S=\frac{d \sigma^{+} / d \phi-d \sigma^{-} / d \phi}{d \sigma^{+} / d \phi+d \sigma^{-} / d \phi}
$$

where $\phi$ in the proton reset frame is the angle between the plane formes by incoming and scattered electrons and that formed by the virtual photon and the scattered proton. $d \sigma^{+} / d \phi$ and $d \sigma^{-} / d \phi$ are the differential cross sections of measured in $e^{+} p$ and $e^{-} p$ respectively. The result is presented in Fig.17. The fit to the measured points with a function $p_{1} \cdot \cos (\phi)$ is also displayed, which gives for $p_{1}$ the value $0.17 \pm 0.03$ (stat.) \pm 0.05 (sys.). Thus, a significant non-zero value of beam charge asymmetry is measured.

\section{Conclusions}

Presented here is a subjective selection taken from the wealth of new measurements of exclusive vector meson production processes delivered by $\mathrm{H} 1$ and ZEUS experiments at HERA collider. Much progress has been made over recent years, in the type of studies that can be performed, the precision achieved and in theoretical understand- 

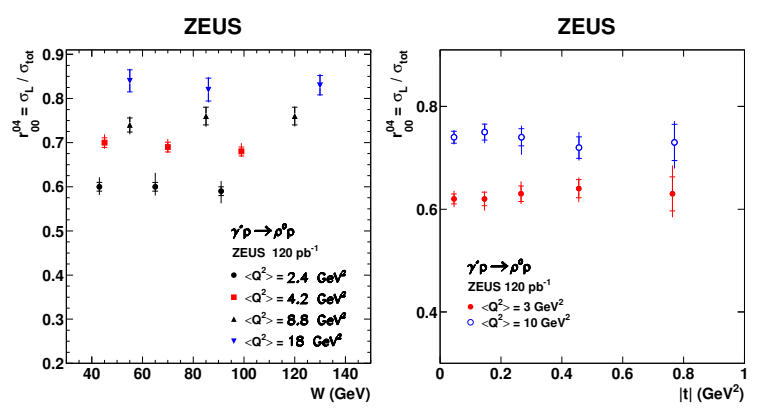

Figure 12. The ratio $r_{00}^{04}=\sigma_{L} / \sigma_{t o t}$ as a function of $W$ and $t$ for different values of $Q^{2}$.
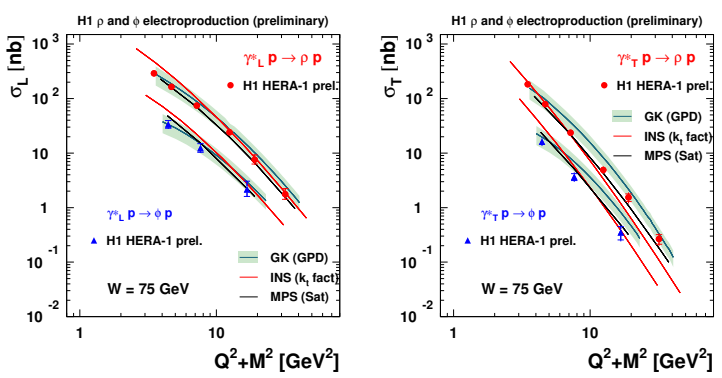

Figure 13. $\left(Q^{2}+M^{2}\right)$ dependences of a longitudinal and transverse $\gamma^{*} p$ cross sections for $\rho$ and $\phi$ meson production with $W=75 \mathrm{GeV}$. The predictions of some selected theory calculations [19-21] are compared to the measurements.

ing. The high precision of the measurements can be used to refine the models and contribute to a better understanding of the vector meson wave functions and of the gluon density in the proton.

\section{Acknowledgments}

I would like to thank organizers for this interesting, stimulating and enjoyable workshop.

\section{REFERENCES}

1. A. Donnachie and P.V. Landshoff, Phys. Lett. B 296 (1992) 227

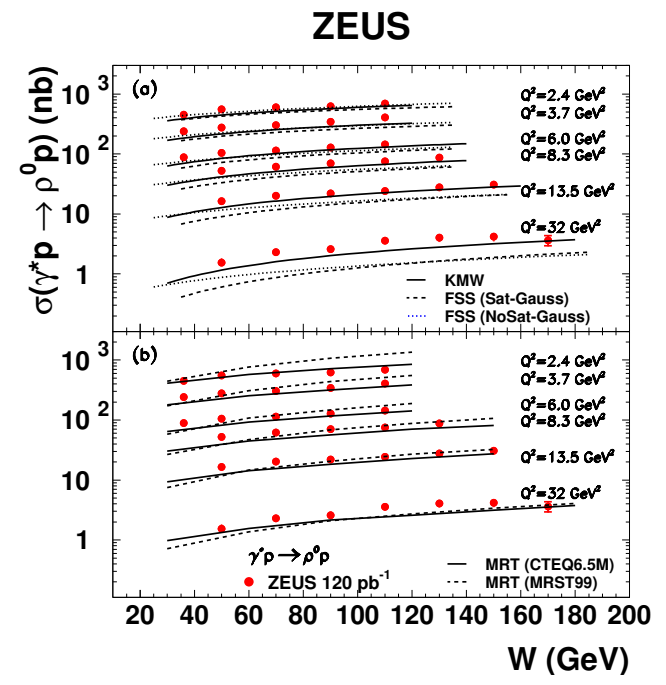

Figure 14. The $W$ dependence of the $\gamma^{*} p \rightarrow \rho^{0} p$ cross section for different values of $Q^{2}$. The predictions of some selected theory calculations $[22,24,25]$ are compared to the measurements.

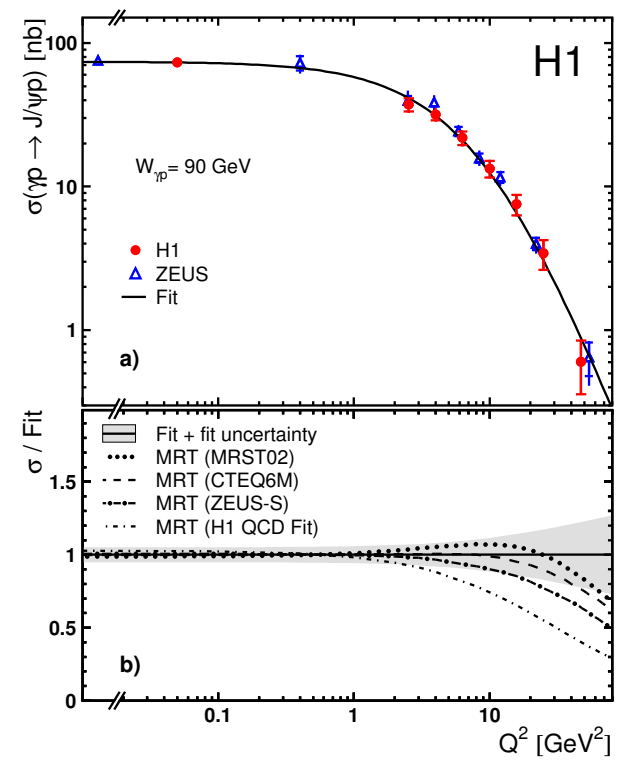

Figure 15. Total cross section for elastic $J / \psi$ electroproduction as a function of $Q^{2}$ in the range $|t|<1.2 \mathrm{GeV}^{2}$. The predictions of MRT theory calculations [22] using the different parameterisations of proton parton densities are compared to the measurements. 


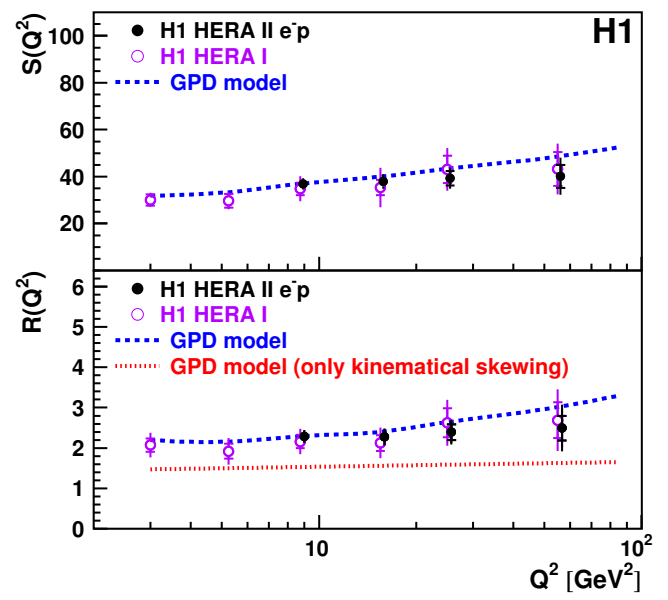

Figure 16. The observables $S$ and $R$, shown as a function of $Q^{2}$. The dashed curves show the predictions of the GPD model $[26,23]$. The dotted curve shows the prediction of a GPD model based on an approximation where only the kinematical part of the skewing effects are taken into account.

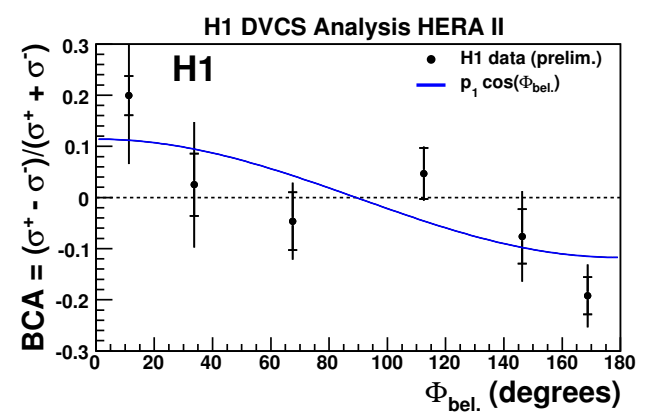

Figure 17. Beam charge asymmetry for DVCS process $\gamma^{*} p \rightarrow \gamma p$ as a function of $\phi$, measured in the range $0.05<|t|<1 \mathrm{GeV}^{2}$. The line represents the result of a $p 1 \cdot \cos (\phi)$ fit to the data points.
2. H1 Collab., Nucl. Phys. B 463 (1996) 3;

3. H1 Collab., Eur. Phys. J. C 46 (2006) 585; Nucl. Phys. B 472 (1996) 3;

4. H1 Collab., Phys. Lett. B 541 (2002) 251;

5. H1 Collab., Phys. Lett. B 483 (2000) 23;

6. ZEUS Collab., Eur. Phys. J. C 6 (1999) 603; Eur. Phys. J. C 2 (1998) 247;

7. ZEUS Collab., Phys. Lett. B 377 (1996) 259;

8. ZEUS Collab., Z. Phys. C 73 (1996) 73;

9. ZEUS Collab., Z. Phys. C 75 (1997) 215; Eur. Phys. J. C 24 (2002) 345;

10. ZEUS Collab., "Exclusive Photoproduction of Upsilon mesons at HERA", Presented at EPS2007, Manchester 2007;

11. ZEUS Collab., Nucl. Phys. B 627 (2002) 3;

12. H1 Collab., Z. Phys. C 69 (1995) 27;

13. ZEUS Collab., PMC Physics A 2007, 1;

14. H1 Collab., "Diffractive rho and phi production in DIS with the $\mathrm{H} 1$ detector", Presented at DIS2008, London 2008; Phys. Lett. B 539 (2002) 25;Eur. Phys. J. C 13 (2000) 371;

15. ZEUS Collab., Nucl. Phys. B 695 (2004) 3;

16. H1 Collab., Phys. Lett. B 659 (2008) 796; Eur. Phys. J. C 44 (2005) 1;

17. ZEUS Collab., "Deeply Virtual Compton Scattering with a Proton Tag", Presented at EPS2007, Manchester 2007; Phys. Lett. B 573 (2003) 46;

18. ZEUS Collab., Eur. Phys. J. C 14 (2000) 213;

19. S.V. Goloskokov and P. Kroll, Eur. Phys. J. C 42 (2005) 281;

20. I.P. Ivanov, N.N. Nikolaev and A.A. Savin, Phys. Part. Nucl. 37 (2006) 1;

21. C. Marquet, R. Peschanski and G. Soyez, Phys. Rev. D 76 (2007) 034011;

22. A.D. Martin, M.G. Ryskin and T. Teubner, Phys. Rev. D 62 (2000) 014022;

23. J. Pumplin et al.,JHEP 0207 (2002) 012;

24. J. Kowalski, L. Motyka and G. Watt, Phys. Rev. D 74 (2006) 074016;

25. J.R. Forshaw, R. Sandapen and G. Shaw, Phys. Rev. D 69 (2004) 094013;

26. A. Freund, Phys. Rev. D 68 (2003) 096006; 